\title{
General Psychiatry Online single-session interventions for Kenyan adolescents: study protocol for a comparative effectiveness randomised controlled trial
}

\author{
Akash R Wasil (D) ,1,2 Tom Lee Osborn (D) ,2,3 John R Weisz, ${ }^{2,3}$ Robert J DeRubeis ${ }^{1}$
}

To cite: Wasil AR, Osborn TL, Weisz JR, et al. Online singlesession interventions for Kenyan adolescents: study protocol for a comparative effectiveness randomised controlled trial. General Psychiatry 2021;34:e100446. doi:10.1136/ gpsych-2020-100446

Received 17 November 2020 Accepted 21 May 2021

Check for updates

(C) Author(s) (or their employer(s)) 2021. Re-use permitted under CC BY-NC. No commercial re-use. See rights and permissions. Published by BMJ.

${ }^{1}$ Department of Psychology, University of Pennsylvania, Philadelphia, Pennsylvania, USA

${ }^{2}$ Shamiri Institute, Nairobi, Kenya

${ }^{3}$ Department of Psychology,

Harvard University, Cambridge,

Massachusetts, USA

Correspondence to

Akash R Wasil;

akashwasil133@gmail.com

\section{ABSTRACT}

Background Mental health problems are the leading cause of disability among adolescents worldwide, yet access to treatment is limited. Brief digital interventions have been shown to improve youth mental health, but little is known about which digital interventions are most effective.

Aims To evaluate the effectiveness of two digital single-session interventions (Shamiri-Digital and DigitalCBT (cognitive-behavioural therapy)) among Kenyan adolescents.

Methods We will perform a school-based comparative effectiveness randomised controlled trial. Approximately 926 Kenyan adolescents will be randomly assigned to one of three conditions: Shamiri-Digital (focused on gratitude, growth mindsets and values), Digital-CBT (focused on behavioural activation, cognitive restructuring and problem solving) or a study-skills control condition (focused on note-taking and essay writing skills). The primary outcomes include depressive symptoms (measured by the Patient Health Questionnaire-8), anxiety symptoms (Generalized Anxiety Disorder Screener-7) and subjective well-being (Short Warwick-Edinburgh Mental Well-being Scale). The secondary outcomes include acceptability, appropriateness, primary control and secondary control. Acceptability and appropriateness will be measured immediately post-intervention; other outcomes will be measured 2 weeks, 4 weeks and 12 weeks postintervention.

Results We hypothesise that adolescents assigned to Shamiri-Digital and adolescents assigned to DigitalCBT will experience greater improvements (assessed via hierarchical linear models) than those assigned to the control group. We will also compare Shamiri-Digital with Digital-CBT, although we do not have a preplanned hypothesis.

Conclusions Our findings will help us evaluate two digital single-session interventions with different theoretical foundations. If effective, such interventions could be disseminated to reduce the public health burden of common mental health problems.

Trial registration number PACTR202011691886690.

\section{INTRODUCTION}

Common mental health problems-such as symptoms of depression and anxiety-are
Strengths and limitations of this study

- Strengths of this study include a large sample size, the deployment of brief and scalable interventions, a comparison between two active interventions, and the real-world implementation of these interventions in schools.

- Limitations of this study include a reliance on selfreport data to assess outcomes and the lack of a notreatment or wait-list control condition (limiting our ability to estimate the potential impact of our study skills control condition)

the leading cause of disability in adolescents, contributing $45 \%$ to the overall burden of disease among young people. ${ }^{1}$ However, only a small fraction of those in need receive any treatment. Even in high-income countries such as the USA, about $65 \%$ of the youth with psychiatric disorders do not receive treatment; among those who do, 30\%-60\% drop out prematurely. ${ }^{23}$ Access to treatment is especially poor in low-income and middleincome countries. ${ }^{4}$ Common barriers to services include high costs of treatment, lack of trained mental health professionals, transportation and scheduling difficulties and stigma associated with professional helpseeking. ${ }^{45}$

This challenge is commonly referred to as the treatment gap - a gap between those with mental health problems and those who receive treatment. ${ }^{6}$ Currently, most empirically supported interventions for the youth are face-to-face interventions that last multiple months. ${ }^{78}$ Although these interventions generally yield medium effects, their implementation often requires substantial time and resources. ${ }^{8}$ To address the treatment gap, experts have called for scalable treatment delivery formats such as digital interventions, self-help interventions and low-intensity prevention interventions. ${ }^{69}$ 
One promising strategy to reduce the treatment gap involves the development and testing of brief, efficient and digital mental health interventions. A growing body of evidence suggests that digital interventions, such as those delivered as web-based programmes, are effective for a variety of mental health conditions. ${ }^{10}{ }^{11}$ Furthermore, meta-analytic evidence suggests that online interventions can be effective in just a single session. ${ }^{12}$ As an example, some self-administered, online singlesession interventions centred around growth mindsets have been shown to reduce depressive symptoms even at the follow-ups at 4-9 months. ${ }^{13} 14$ Such interventions could be disseminated widely at low costs, making them particularly promising candidates as scalable global mental health interventions. ${ }^{15} 16$ However, most of the researches on single-session interventions have been conducted in Western countries, and little is known about the potential effectiveness and acceptability of these interventions in non-Western settings. Our team previously conducted one of the first pilot trials of an online single-session intervention in Sub-Saharan Africa with Kenyan adolescents. In that trial, participants randomised to Shamiri-Digital-an intervention that included lessons on growth mindsets, gratitude and value affirmations-experienced greater reductions in depression and anxiety than those randomised to a study-skills control condition. ${ }^{17}$ While this work suggests that single-session interventions have potential, follow-up work with larger sample sizes and longer follow-up time points is needed.

Additionally, little is known about which content is best suited for brief digital interventions. While previous research has shown that active interventions outperform control groups ${ }^{12}$, it remains unclear if certain active interventions are more effective than others. Understanding which single-session interventions are most effective is especially important in low-resource settings, where investment in mental health interventions is poor. Such research can benefit policymakers and practitioners who could prioritise limited resources to disseminate the most impactful interventions.

Guided by this logic, we are performing a randomised controlled trial (RCT) comparing two active single-session interventions against each other and against an active control group. The first intervention, Shamiri-Digital, will be derived from our team's previous work with Kenyan adolescents. ${ }^{17}$ The second intervention, Digital-CBT, will be adapted from traditional cognitive-behavioural therapy (CBT) exercises as well as our team's previous work in India and the USA. ${ }^{18} 19$ The active control group will consist of online study-skills exercises that have been used previously in RCTs with Kenyan youths. ${ }^{17}$

Our trial has four aims. The first is to test whether Shamiri-Digital can effectively reduce depressive and anxiety symptoms among Kenyan adolescents. We hypothesise that adolescents assigned to Shamiri-Digital condition will experience larger symptom reductions than those assigned to an active study-skills control group.
The second aim is to evaluate the efficacy of a singlesession intervention based on CBT exercises. Previous meta-analytic research has shown that digital CBT interventions are effective for depression and anxiety. ${ }^{11}{ }^{20}$ Notably, trials from these meta-analyses have generally tested digital CBT interventions that last multiple months. In contrast, limited number of researches have examined the effects of single-session interventions grounded in CBT. In this study, we hypothesise that adolescents assigned to the Digital-CBT condition will experience larger symptom reductions than those assigned to the study-skills control group.

Our third aim is to compare the effects of ShamiriDigital and Digital-CBT. Although there are many trials of digital CBT interventions, we are unaware of any digital CBT interventions that have been tested specifically for Kenyan youths. In contrast, there has been only one trial of Shamiri-Digital, but this trial was conducted with Kenyan youths and produced medium-to-large effects on youth depressive symptoms. ${ }^{17}$ Thus, Digital-CBT has a broader evidence base, but Shamiri-Digital has been tested specifically with Kenyan youths. As a result, we do not have a hypothesis regarding which intervention will be more effective. For each intervention, we expect that the effect will be stronger at 2-week follow-up than at 4-week or 12-week follow-up.

Our fourth aim is to examine the acceptability and appropriateness of each intervention. We hypothesise that each intervention will receive adequate acceptability and appropriateness ratings. Through this aim, we hope to identify which interventions Kenyan adolescents perceive to be appealing and helpful.

\section{METHODS}

\section{Study design}

Trial design

This will be a double-blind RCT with three arms: ShamiriDigital, Digital-CBT and a study-skills control intervention (see figure 1). Interventions will be delivered in schools' computer laboratories in groups of 10-30 students. Each student will be assigned to a private computer. Assessments will be administered at baseline and at 2-week, 4-week and 12-week follow-up. The trial has also been preregistered at the Pan African Clinical Trials Registry in accordance with WHO and International Committee of Medical Journal Editors standards.

\section{Trial setting}

The study will take place in secondary schools located in Nairobi and neighbouring counties in Kenya. The study team will identify the schools through the Shamiri Institute, a local non-profit in Kenya; we plan to recruit two to three schools. Principals of the schools will then be contacted and provided with information about the study, and those wishing to enrol their schools signed up. If we receive interest from officials at more than two to three schools, we will choose the final participating schools with 

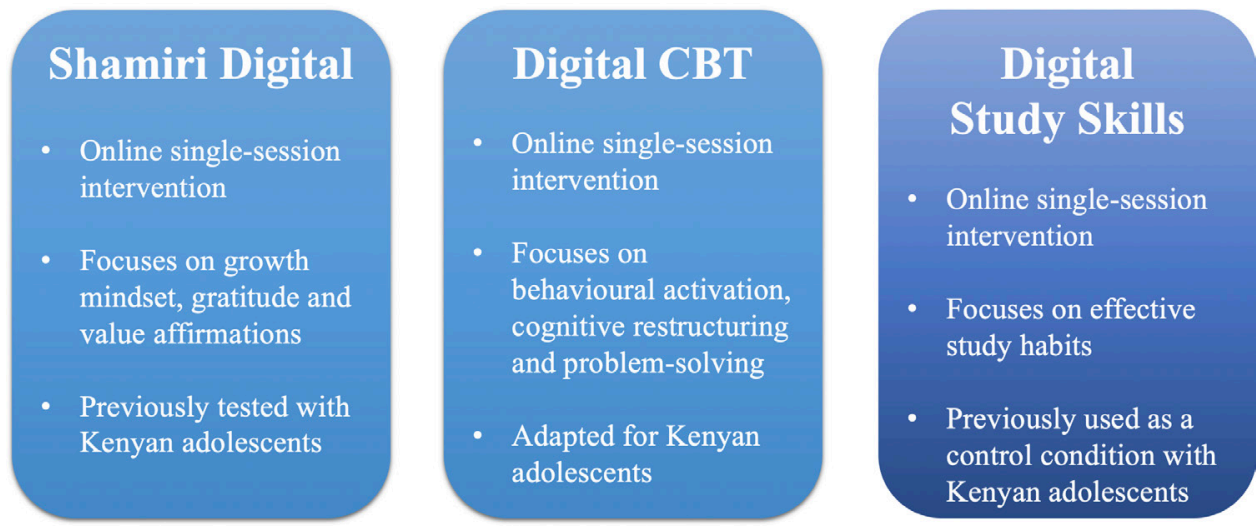

Figure 1 Summary of the two intervention conditions (Shamiri-Digital and Digital-CBT) and the study-skills control condition. All three conditions will be administered online via a single session. CBT, cognitive-behavioural therapy.

an eye towards ensuring sociocultural and sociodemographic diversity of the final sample, as has been outlined elsewhere. $^{2122}$

\section{Participant recruitment}

In all participating schools, students in form 1, form 2, form 3 and form 4 (ie, grades 9-12) will be notified of the study by school administrators per local school customs. All students aged between 13 and 18 inclusive will be eligible. Because our aim is to evaluate the interventions as universal interventions, no exclusion criteria will be applied. Parental consents will be sought for all minor participants per institutional review board regulations. All students will provide either informed consents or assents if they are minors.

\section{Randomisation and treatment allocation}

Study activities will be conducted in computer laboratories in the participating Kenyan secondary schools. Because the computer laboratories have between 10 and 30 computers, the study activities will be conducted in groups of between 10 and 30 students. At the start of each session, participants will be briefed by study staff on study activities, how to get help should they need it and that they can stop study activities at any time. Each participant will then be randomised to one of the three study conditions using a random number generator embedded in the study website.

\section{Procedure}

Participants will begin by reading information about the study and the consent form. They will then fill out the baseline measures. After this, participants will be randomised to one of the three conditions (see figure 1). Finally, they will complete a sociodemographic and feasibility/acceptability measure. In total, participants will take approximately $90 \mathrm{~min}$ to complete these steps: $30 \mathrm{~min}$ for questionnaires and $60 \mathrm{~min}$ for intervention exercises. Participants will complete follow-up measures at 2 weeks, 4 weeks and 12 weeks after study activities. To reduce dropout rates during follow-up, participants will be entered into a lottery to receive 500 Kenyan shilling (US\$5) and a T-shirt; these strategies have been used in other studies with Kenyan adolescents. ${ }^{1722}$

\section{Timeline for study and data collection}

Participant recruitment will occur from May 2021 to July 2021. Follow-up data collection will end in October 2021, once final follow-up measures are collected. We intend to report the results by May 2022. On termination of data collection and publication of results, we will make de-identified study data publicly accessible.

\section{Intervention arms}

Shamiri-Digital

The Shamiri-Digital intervention will be adapted from a previous study with Kenyan youths. ${ }^{17}$ The intervention consists of three modules: growth mindset, gratitude and value affirmations. The growth mindset activities will teach participants about the brain's ability to grow in response to difficulties/challenges in various domains (eg, interpersonal relationships, personality traits and academic performance). They will also write 'growth stories', in which they reflect on a challenge that they have faced and how they overcame that challenge. In the gratitude exercises, participants will learn about gratitude and the importance of practising and expressing gratitude. Through a 'good things' exercise, participants will list three good things in their lives for which they are grateful. Finally, in the value affirmation exercises, participants will reflect on values that are important to them and brainstorm ways to apply these values in new ways.

\section{Digital-CBT}

The Digital-CBT intervention will be adapted from previous work adapting exercises from CBT for digital single-session interventions. ${ }^{18} 19$ The intervention will consist of three modules: cognitive restructuring, problem solving and behavioural activation. The cognitive restructuring activities will teach participants to 
identify and reframe negative thoughts. Participants will read testimonials about a Kenyan peer who is experiencing negative thoughts about a stressful situation. Participants will then identify negative beliefs that the student is facing and generate ways that the student could reframe those beliefs. Then, participants will apply the same cognitive restructuring technique to a situation in their own lives. ${ }^{19}$ In the problem solving exercise, participants will learn an evidence-based approach to problem solving involving five steps: (1) identifying and defining a problem, (2) generating a list of possible solutions, (3) evaluating the strengths and weaknesses of the possible solutions, (4) selecting a solution and (5) applying the solution and determining whether an additional solution is needed. ${ }^{23}$ Participants will be instructed to think of a problem they are currently facing and then apply the aforementioned problem solving method. In the behavioural activation exercise, participants will identify pleasurable activities and mastery activities and reflect on why these activities are important to them. Then, they will complete a 'positive activity plan' in which they identify an activity they want to perform more in the upcoming weeks, list when and how they will perform the activity and brainstorm ways they can overcome potential obstacles. ${ }^{19}$

\section{Study-skills control}

The content for the study-skills control will be adapted from a previous study-skills control intervention that has been used in a previous RCT of Shamiri-Digital. ${ }^{17}$ The structure of the study-skills activities will mirror the structure of Shamiri-Digital and Digital-CBT and will require similar effort and time.

\section{Measures}

\section{Primary outcomes}

Depressive symptoms will be assessed using the Patient Health Questionnaire-8 (PHQ-8). This is the eight-item version of PHQ-9, which is a brief diagnostic and severity measure for depression. ${ }^{24}$ The PHQ-8 has been used in previous studies with Kenyan youths ${ }^{21}{ }^{22}$, including the previous trial of Shamiri-Digital. ${ }^{17}$ The PHQ-8 has demonstrated robust psychometric measures with Kenyan adolescents. ${ }^{21} 25$

Anxiety symptoms will be assessed using the Generalized Anxiety Disorder Screener (GAD-7). The GAD-7 has shown adequate internal consistency and validity in Western samples. ${ }^{26}$ The GAD-7 has also demonstrated robust psychometric measures with Kenyan adolescents. $^{21} 25$

Subjective well-being will be assessed using the Short Warwick-Edinburgh Mental Well-being Scale. ${ }^{27}$ The scale consists of seven positively worded statements, including 'I've been dealing with problems well' and 'I've been feeling useful'. It has demonstrated adequate internal consistency and validity in Western samples ${ }^{27}$ and has been used in a previous trial of Shamiri-Digital. ${ }^{19}$

\section{Secondary outcomes}

Acceptability (ie, the extent to which a given treatment is agreeable or satisfactory) and appropriateness (ie, the extent to which a treatment is perceived as relevant or compatible in a given context) will be assessed using the Acceptability of Intervention Measure (AIM) and the Intervention Appropriateness Measure (IAM) ${ }^{28}$ AIM and IAM have demonstrated strong internal consistency, structural validity and content validity. ${ }^{28}$ To our knowledge, this is the first time they will be administered to Kenyan adolescents.

Primary control (ie, the extent to which individuals believe they can affect objective conditions in their lives, such as their grades or the actions of other people) will be assessed using items from the Perceived Control Scale for Children (PCSC).$^{29}$ Secondary control (ie, the extent to which individuals believe that they can manage the personal and psychological impact of objective events) will be assessed using items from the Secondary Control Scale for Children (SCSC).${ }^{28}$ Low primary control and low secondary control are both considered risk factors for mental health problems in youth. ${ }^{29}$ The PCSC is a 24 -item measure and the SCSC is a 20-item measure; to reduce participant fatigue, we will select a subset of the items from each measure.

\section{Data analysis plan}

Sample size and statistical power

We intend to recruit 926 adolescents. An optimal design was used to calculate the sample size needed to achieve a power of 0.8 with an alpha level of 0.0167 ( 0.05 divided by 3 , to account for multiple comparisons). We powered to detect a modest standardised effect of $d=0.30$, yielding a required sample size of 741 . Given the scalability and low cost of the interventions, even 'small' effects are worth detecting. Low-intensity interventions with small effects can have meaningful public health impacts given their potential to reach a large number of people. Assuming an attrition rate of 20\%, 926 participants will be required.

\section{Analysis of primary outcomes}

We will conduct mixed-effects linear models to analyse changes in depressive symptoms, anxiety symptoms and subjective well-being. For each of the three dependent variables (PHQ-8 symptoms, GAD-7 symptoms and Warwick well-being scores), we will perform two models. One model will include a random intercept for each subject (accounting for variation between individuals at baseline) and a random intercept for each school. The second model will include these random intercepts as well as a random slope (accounting for variation at baseline and variation in symptom changes over time). For both models, we will include time, condition and the time*condition interaction as independent variables; we will also include gender and age as covariates. Because the study includes three conditions, we plan to conduct and report three comparisons: (1) Shamiri-Digital versus control, (2) Digital-CBT versus control and (3) Shamiri-Digital versus 
Digital-CBT. We will apply the Holm-Bonferroni correction for multiple comparisons.

For each dependent variable, we will compare the first model (random intercepts only) and the second model (random intercepts and a random slope) using Akaike information criterion (AIC) values. The AIC method assesses model fit while penalising models with larger numbers of parameters. We will report the results of the models with better (ie, lower) AIC values. Models will be automatically excluded if they failed to converge. We will also evaluate models in which gender and age are included as covariates. Furthermore, we will calculate standardised effect sizes (Cohen's $d$ ) for each comparison. We will use mean change scores, consistent with the method we applied in our previous trial of Shamiri-Digital. ${ }^{17}$

In addition to examining the effect of the interventions on the complete sample, we will evaluate the effect of the interventions on the sub-sample of adolescents with clinically elevated symptoms at baseline. To identify this sub-sample, we will apply the conventional clinical cut-offs, defined as a score greater than or equal to 10 on either the PHQ-8 or the GAD-7 ${ }^{24}{ }^{26}$ We will perform the same analyses described above on this sub-sample of participants.

Missing item-level data (eg, one question on a questionnaire) will be imputed five times using the fully conditional specification procedure using the multivariate imputation by chained equations (mice) function in $\mathrm{R}$. We will also report the rates of attrition and the percentage of participants with missing item-level data.

\section{Analysis of secondary outcomes}

Acceptability will be measured immediately postintervention. To examine differences in acceptability between the three conditions, we will perform a one-way analysis of variance. If this omnibus test is statistically significant $(\mathrm{p}<0.05)$, we will conduct follow-up t-tests to examine the differences in acceptability between each pair of conditions.

Primary and secondary control will be measured preintervention and post-intervention. To examine pre-post changes for each form of control, we will perform hierarchical linear models using the same approach described above for our primary outcome measures.

\section{DISCUSSION}

The objective of this RCT is to evaluate two digital single-session interventions for adolescent mental health promotion. The two interventions differ considerably in their content-with one focusing on traditional CBT techniques and the other focusing on character strengths. Secondary aims include examining the acceptability, perceived utility and pre-post impact of each intervention. Potential challenges include school recruitment and student retention. To address these challenges, we will leverage existing partnerships through the Shamiri Institute (https://www.shamiri.institute/) and apply best practices we have learnt through our previous work in Kenya. $^{30} 31$

Our results will indicate whether these scalable online single-session interventions have the potential to improve adolescent mental health and well-being in Kenya. Given the burden of mental health problems among the youth in Sub-Saharan Africa and the lack of treatment options, there is a great need for scalable mental health interventions. Results of this trial will help us understand if scalable single-session interventions could serve as one tool to reduce the burden of mental health problems among the youth in Kenya. Future work could examine these single-session interventions in other low-income and middle-income countries where adolescent mental health problems are common and undertreated..$^{32} 33$

Twitter Akash R Wasil @AkashWasil and Tom Lee 0sborn @tomleeosborn

Contributors AW and TLO designed the study with guidance from JRW and RD. AW, TLO, JRW and RD participated in drafting and revising the manuscript.

Funding The authors have not declared a specific grant for this research from any funding agency in the public, commercial or not-for-profit sectors.

Competing interests TLO, AW and JRW are affiliated with the Shamiri Institute.

Patient consent for publication Not required.

Ethics approval Study methods and protocols have been approved by the University of Pennsylvania Institutional Review Board. We will also receive ethical approval from a local Kenyan university ethics review committee and obtain a research permit from the National Commission for Science, Technology and Innovation before starting study activities.

Provenance and peer review Not commissioned; externally peer reviewed.

Data availability statement There are no data in this work.

Open access This is an open access article distributed in accordance with the Creative Commons Attribution Non Commercial (CC BY-NC 4.0) license, which permits others to distribute, remix, adapt, build upon this work non-commercially, and license their derivative works on different terms, provided the original work is properly cited, appropriate credit is given, any changes made indicated, and the use is non-commercial. See: http://creativecommons.org/licenses/by-nc/4.0/.

ORCID iDs

Akash R Wasil http://orcid.org/0000-0002-0326-0364

Tom Lee Osborn http://orcid.org/0000-0002-9092-1035

\section{REFERENCES}

1 Gore FM, Bloem PJN, Patton GC, et al. Global burden of disease in young people aged 10-24 years: a systematic analysis. Lancet 2011;377:2093-102.

2 Harpaz-Rotem I, Leslie D, Rosenheck RA. Treatment retention among children entering a new episode of mental health care. Psychiatr Serv 2004;55:1022-8.

3 Merikangas KR, He J-ping, Burstein M, et al. Service utilization for lifetime mental disorders in U.S. adolescents: results of the National comorbidity Survey-Adolescent supplement (NCS-A). J Am Acad Child Adolesc Psychiatry 2011;50:32-45.

4 Bruckner TA, Scheffler RM, Shen G, et al. The mental health workforce gap in low- and middle-income countries: a needs-based approach. Bull World Health Organ 2011;89:184-94.

5 Patel V, Flisher AJ, Hetrick S, et al. Mental health of young people: a global public-health challenge. Lancet 2007;369:1302-13.

6 Kazdin AE. Addressing the treatment gap: a key challenge for extending evidence-based psychosocial interventions. Behav Res Ther 2017;88:7-18.

7 Southam-Gerow MA, Prinstein MJ. Evidence base updates: the evolution of the evaluation of psychological treatments for children and adolescents. J Clin Child Adolesc Psychol 2014;43:1-6.

8 Weisz JR, Kuppens S, Ng MY, et al. What five decades of research tells us about the effects of youth psychological therapy: a multilevel 
meta-analysis and implications for science and practice. Am Psychol 2017;72:79-117.

9 Fairburn CG, Patel V. The impact of digital technology on psychological treatments and their dissemination. Behav Res Ther 2017;88:19-25.

10 Andrews G, Basu A, Cuijpers P, et al. Computer therapy for the anxiety and depression disorders is effective, acceptable and practical health care: an updated meta-analysis. $J$ Anxiety Disord 2018;55:70-8.

11 Ebert DD, Zarski A-C, Christensen H, et al. Internet and computerbased cognitive behavioral therapy for anxiety and depression in youth: a meta-analysis of randomized controlled outcome trials. PLoS One 2015;10:e0119895.

12 Schleider JL, Weisz JR. Little Treatments, Promising Effects? Meta-Analysis of Single-Session Interventions for Youth Psychiatric Problems. J Am Acad Child Adolesc Psychiatry 2017;56:107-15.

13 Miu AS, Yeager DS. Preventing symptoms of depression by teaching adolescents that people can change: effects of a brief incremental theory of personality intervention at 9-month follow-up. Clinical Psychological Science 2015;3:726-43.

14 Schleider J, Weisz J. A single-session growth mindset intervention for adolescent anxiety and depression: 9-month outcomes of a randomized trial. J Child Psychol Psychiatry 2018;59:160-70.

15 Hall CS, Fottrell E, Wilkinson S, et al. Assessing the impact of mHealth interventions in low- and middle-income countries--what has been shown to work? Glob Health Action 2014;7:25606.

16 Muñoz RF. Using evidence-based Internet interventions to reduce health disparities worldwide. J Med Internet Res 2010;12:e60

17 Osborn TL, Rodriguez M, Wasil AR, et al. Single-session digital intervention for adolescent depression, anxiety, and well-being: outcomes of a randomized controlled trial with Kenyan adolescents. J Consult Clin Psychol 2020;88:657-68.

18 Wasil AR, Park SJ, Gillespie S, et al. Harnessing single-session interventions to improve adolescent mental health and well-being in India: development, adaptation, and pilot testing of online singlesession interventions in Indian secondary schools. Asian J Psychiatr 2020;50:101980.

19 Wasil AR, Taylor ME, Franzen RE, et al. Promoting graduate student mental health during COVID-19: acceptability, feasibility, and perceived utility of an online Single-Session intervention. Front Psychol 2021;12:569785.

20 Karyotaki E, Riper H, Twisk J, et al. Efficacy of Self-guided Internetbased cognitive behavioral therapy in the treatment of depressive symptoms: a meta-analysis of individual participant data. JAMA Psychiatry 2017;74:351-9.

21 Osborn TL, Venturo-Conerly KE, Wasil AR, et al. Depression and anxiety symptoms, social support, and demographic factors among Kenyan high school students. J Child Fam Stud 2020;29:1432-43.

22 Osborn TL, Wasil AR, Venturo-Conerly KE, et al. Group intervention for adolescent anxiety and depression: outcomes of a randomized trial with adolescents in Kenya. Behav Ther 2020;51:601-15.

23 Ugueto AM, Santucci LC, Krumholz LS, et al. Problemsolving skills training. Evidence-based CBT for anxiety and depression in children and adolescents: A competencies-based approach:247-59.

24 Kroenke K, Spitzer RL. The PHQ-9: a new depression diagnostic and severity measure. Psychiatr Ann 2002;32:509-15.

25 Osborn TL, Venturo-Conerly KE, Gan JY, et al. Depression and anxiety symptoms amongst Kenyan adolescents: psychometric properties, prevalence, psychosocial and sociodemographic factors. PsyArXiv 2021.

26 Spitzer RL, Kroenke K, Williams JBW, et al. A brief measure for assessing generalized anxiety disorder: the GAD-7. Arch Intern Med 2006;166:1092-7.

27 Tennant R, Hiller L, Fishwick R, et al. The Warwick-Edinburgh mental well-being scale (WEMWBS): development and UK validation. Health Qual Life Outcomes 2007;5:63.

28 Weiner BJ, Lewis CC, Stanick C, et al. Psychometric assessment of three newly developed implementation outcome measures. Implement Sci 2017;12:108.

29 Weisz JR, Francis SE, Bearman SK. Assessing secondary contro and its association with youth depression symptoms. J Abnorm Child Psychol 2010;38:883-93.

30 Wasil AR, Osborn TL, Venturo-Conerly KE, et al. Conducting global mental health research: lessons learned from Kenya. Glob Ment Health 2021;8:1-4.

31 Osborn TL, Wasil AR, Weisz JR, et al. Where is the global in global mental health? A call for inclusive multicultural collaboration. Gen Psychiatr 2020;33:e100351.

$32 \mathrm{Xu}$ Y. New journey, new mission: bolstering international communication about mental health. Gen Psychiatr 2018;31:e100002.

33 Zhang F, Cloutier PF, Yang H, et al. Non-suicidal self-injury in Shanghai inner bound middle school students. Gen Psychiatr 2019;32:e100083.

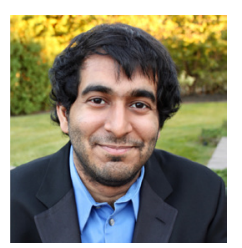

Akash Wasil graduated Phi Beta Kappa from Harvard University, where he served as a peer counselor with Harvard ECHO (Eating Concerns Hotline and Outreach) and performed research with the Weisz Lab for Youth Mental Health. At present, he is a second-year PhD student and National Science Foundation research fellow in clinical psychology working with Robert DeRubeis at the University of Pennsylvania in the United States. Driven by a desire to expand access to evidence-based treatments, Akash's research focuses on developing and evaluating scalable mental health interventions for vulnerable populations. He is especially interested in digital mental health, global mental health, implementation science, health economics and effective altruism. Akash has experience evaluating smartphone applications for common mental health problems, designing novel digital mental health interventions and working with multicultural teams to adapt interventions for people in Kenya, India, Greece and the United States. 